\title{
Asymptotic behavior of minima and mountain pass solutions for a class of Allen-Cahn models
}

\author{
Jaeyoung Byeon and Paul H. Rabinowitz
}

In an earlier paper, the authors studied a class of Allen-Cahn models for which the solution was near 1 on a prescribed set, $T+[0,1]^{n}$ where $T \subset \mathbb{Z}^{n}$, and near 0 on its complement. In this note, when $T$ is finite and consists of two widely spaced subsets, $T_{1}$ and $l+T_{2}$ with $l \in \mathbb{Z}^{n}$, we study the asymptotic behavior of two special families of solutions as $l \rightarrow \infty$.

\section{Introduction}

In two recent papers [9], [10], the authors studied an Allen-Cahn model problem having the form

$$
-\Delta u+A_{\varepsilon}(x) G^{\prime}(u)=0, \quad x \in \mathbb{R}^{n}
$$

where $G(u)=u^{2}(1-u)^{2}$ is a double well potential, $\varepsilon>0$, and $A_{\varepsilon}(x)=$ $1+A(x) / \varepsilon$ with $0 \leq A \in C^{1}\left(\mathbb{R}^{n}\right), 1$-periodic in $x_{1}, \cdots, x_{n}, \Omega$ is the support of $\left.A\right|_{[0,1]^{n}}$ and has a smooth boundary, and $\bar{\Omega} \subset(0,1)^{n}$. A main result of [9] is that there is an $\varepsilon_{0}>0$ such that for any finite set $T \subset \mathbb{Z}^{n}$ and $\varepsilon \in\left(0, \varepsilon_{0}\right]$, (1.1) has a solution, $U_{\varepsilon, T}$ with $0<U_{\varepsilon, T}<1, U_{\varepsilon, T}$ is near 1 on $A^{T} \equiv T+\bar{\Omega}$ and near 0 on $B^{T} \equiv\left(\mathbb{Z}^{n} \backslash T\right)+\bar{\Omega}$. Moreover as $\varepsilon \rightarrow 0, U_{\varepsilon, T} \rightarrow 1$ uniformly on $A^{T}$ and $U_{\varepsilon, T} \rightarrow 0$ uniformly on $B^{T}$. When $T$ is finite, $U_{\varepsilon, T}$ is characterized as the minimizer of a constrained variational problem associated with (1.1). Although $U_{\varepsilon, T}$ may not be unique, the set of such minimizers, $\mathcal{M}_{\varepsilon}(T)$, is ordered. The setting of [9] was further treated in [10] where it was shown that for each finite $T$, there is an $\varepsilon_{1}(T)>0$ such that for $\varepsilon \in\left(0, \varepsilon_{1}(T)\right)$, (1.1) has a solution, $V_{\varepsilon, T}$ of mountain pass type with $0<V_{\varepsilon, T}<U_{\varepsilon, T}$.

The main goal of this note is to study the setting of when $T$ is finite and consists of two widely separated subsets, that is, $T=T_{1} \cup\left(l+T_{2}\right) \equiv T_{l}$ for $T_{1}, T_{2} \subset \mathbb{Z}^{n}, l \in \mathbb{Z}^{n}$ and large $|l|>0$. In particular we are interested in the asymptotic behavior as $l \rightarrow \infty$ of the minimizers, $U_{\varepsilon, T_{l}}$, and the mountain pass solutions, as well as the corresponding critical values. To describe our 
results, let

$$
J_{\varepsilon}(u)=\int_{\mathbb{R}^{n}} \frac{1}{2}|\nabla u|^{2}+A_{\varepsilon}(x) G(u) d x,
$$

the functional associated with (1.1). Set

$$
c_{\varepsilon}\left(T_{l}\right)=J_{\varepsilon}\left(U_{\varepsilon, T_{l}}\right)
$$

A more precise characterization of $c_{\varepsilon, T_{l}}$ will be given later. We will prove

Theorem 1.2. Suppose $T \subset \mathbb{Z}^{n}$ is finite. Let $A_{\varepsilon}$ and $G$ be as above. Then for any $\varepsilon \in\left(0, \varepsilon_{0}\right]$, as $l \rightarrow \infty$,

$1^{o} \quad c_{\varepsilon}\left(T_{l}\right) \rightarrow c_{\varepsilon}\left(T_{1}\right)+c_{\varepsilon}\left(T_{2}\right) ;$

$2^{o}$ There is a $U_{\varepsilon, T_{1}} \in \mathcal{M}_{\varepsilon}\left(T_{1}\right)$ such that $U_{\varepsilon, T_{l}} \rightarrow U_{\varepsilon, T_{1}}$ along a subsequence in $C_{l o c}^{2}\left(\mathbb{R}^{n}\right)$;

$3^{o}$ There is a $U_{\varepsilon, T_{2}} \in \mathcal{M}_{\varepsilon}\left(T_{2}\right)$ such that $U_{\varepsilon, T_{l}}(\cdot+l) \rightarrow U_{\varepsilon, T_{2}}$ along a subsequence in $C_{\text {loc }}^{2}\left(\mathbb{R}^{n}\right)$.

Thus, roughly speaking, the minimizer for the $T_{l}$ problem is obtained by gluing translates of the minimizers for the $T_{1}$ and $T_{2}$ problems. These results will be carried out in $\S 2$. Then in $\S 3$, we will give sharper results for the setting of [10] on mountain pass solutions. In particular for large $l$ and small $\varepsilon$, it will be shown that there are two critical values of mountain pass type. One of the associated critical points of $J_{\varepsilon}$ corresponds to gluing a minimum, $U_{\varepsilon, T_{1}}$ of $J_{\varepsilon}$ to a mountain pass solution, $V_{\varepsilon, l+T_{2}}=V_{\varepsilon, T_{2}}(\cdot-l)$, and the other to gluing a $V_{\varepsilon, T_{1}}$ to a $U_{\varepsilon, l+T_{2}}=U_{\varepsilon, T_{2}}(\cdot-l)$. Moreover as $l \rightarrow \infty$, the corresponding critical values converge to the sum of $c_{\varepsilon}\left(T_{1}\right)$ and $J_{\varepsilon}\left(V_{\varepsilon, T_{2}}\right)$, and the sum of $J_{\varepsilon}\left(V_{\varepsilon, T_{1}}\right)$ and $c_{\varepsilon}\left(T_{2}\right)$, respectively. Some final remarks will be made in $\S 4$.

There has been a considerable amount of additional work on solutions of heteroclinic or homoclinic type of Allen-Cahn model equations. See [1]-[4], [12], [16]-[19]. The models involve forcing terms that are periodic in one or all spatial variables with the exception of [4] where there is almost periodic forcing. Aside from [16], minimization arguments are used to obtain solutions of the model equations that are in $C^{2}\left(\mathbb{R} \times \mathbb{T}^{n-1}\right)$ or in $C^{2}\left(\mathbb{R}^{2} \times \mathbb{T}^{n-2}\right)$. In the first case of $C^{2}\left(\mathbb{R} \times \mathbb{T}^{n-1}\right)$, the solutions treated in [12], [17]-[19] are heteroclinic or homoclinic in one direction, say the $x_{1}$-direction, and are periodic in the remaining variables. Moreover the asymptotic states in the $x_{1}$ direction are spatially periodic minimizers of an associated functional. The second case of solutions in $C^{2}\left(\mathbb{R}^{2} \times \mathbb{T}^{n-2}\right)$ is studied in [1]-[4] and [17]-[19]. Here the solutions are heteroclinic in $x_{2}$ between between a pair of $x_{1}$ heteroclinics 
as obtained in the previous case. Solutions of mountain pass type have also been considered in [12]. Although it uses different kinds of arguments based on sub- and supersolutions and comparison arguments, [16] is the only paper aside from [9]-[10] and the current one, which treat solutions other than the heteroclinics or homoclinics in one direction mentioned above.

Using a trick involving the Maximum Principle, see [20], most of the papers mentioned above can be viewed as special cases of a more general class of quasilinear elliptic partial differential equations introduced by Moser in [15]. It's simplest semilinear form is:

$$
-\Delta u+F_{u}(x, u)=0
$$

where $F \in C^{2}\left(\mathbb{T}^{n+1}, \mathbb{R}\right)$. Some papers which study (1.3) in the spirit of the research cited for (1.1) are [5]-[7], [11], [13], [15], [20].

\section{The Proof of Theorem 1.2}

In order to prove Theorem 1.2, some results from [9] must be recalled. In particular, the minimization characterization of $c_{\varepsilon}(T)$ for finite $T$ is required as well as some decay estimates for $U_{\varepsilon, T}$. Thus let $\mathcal{W}$ denote the closure of $C_{0}^{\infty}\left(\mathbb{R}^{n}\right)$ functions under the norm

$$
\|u\| \equiv\left(\int_{\mathbb{R}^{n}}|\nabla u|^{2} d x+\int_{[-1,1]^{n}} u^{2} d x\right)^{1 / 2} .
$$

Let $d^{*}=\frac{1}{2}\left|\partial \Omega-\partial[0,1]^{n}\right|$ and choose any small $d \in\left(0, d^{*}\right)$ so that if

$$
\Omega_{d} \equiv\{x \in \Omega|| x-\partial \Omega \mid>d\},
$$

then $\partial \Omega_{d}$ is diffeomorphic to $\partial \Omega$. For $T \subset \mathbb{Z}^{n}$, set $A_{T}=T+\Omega_{d}$ and $B_{T}=$ $\left(\mathbb{Z}^{n} \backslash T\right)+\Omega_{d}$. Choosing constants $a$ and $b$ so that $0<b<\frac{1}{2}<a<1$ and setting

$$
\Gamma(T)=\left\{u \in \mathcal{W} \mid u \geq a \text { on } A_{T} \text { and } u \leq b \text { on } B_{T}\right\}
$$

define

$$
c_{\varepsilon}(T)=\inf _{u \in \Gamma(T)} J_{\varepsilon}(u) .
$$

Let $\chi_{S}$ denote the characteristic function of the set $S$. Then, as was shown in [9], 
Theorem 2.2. Let $A_{\varepsilon}$ and $G$ be as above. Then there exists an $\varepsilon_{0}>0$ such that for each $\varepsilon \in\left(0, \varepsilon_{0}\right]$ and each finite $T \subset \mathbb{Z}^{n}$,

$1^{o} \mathcal{M}_{\varepsilon}(T) \equiv\left\{u \in \Gamma(T) \mid J_{\varepsilon}(u)=c_{\varepsilon}(T)\right\} \neq \emptyset$.

$2^{o}$ Any $U \in \mathcal{M}_{\varepsilon}(T)$ satisfies $0<U<1$ and is a classical solution of (1.1).

$3^{o} \mathcal{M}_{\varepsilon}(T)$ is an ordered set: $U, V \in \mathcal{M}_{\varepsilon}(T)$ implies $U<V, U>V$, or $U \equiv V$.

$4^{o}$ If $T \subset S \subset \mathbb{Z}^{n}, U_{\varepsilon, T} \leq U_{\varepsilon, S}$ with strict inequality if $T \neq S$.

$5^{o}$ There exist constants $C, c>0$, independent of $T$ and of $\varepsilon \in\left(0, \varepsilon_{0}\right]$, satisfying

$$
\left|U_{\varepsilon, T}(x)-\chi_{T+[0,1]^{n}}(x)\right| \leq C \exp (-c d(x, T)), \quad x \in \mathbb{R}^{n}
$$

where $d(x, T) \equiv \operatorname{dist}\left(x, \partial\left(T+[0,1]^{n}\right)\right)$.

Now with the aid of these preliminaries, we can give the

Proof of Theorem 1.2: There is a positive integer $m$ such that $T_{i}+[0,1]^{n} \subset$ $[-m, m]^{n}$ for $i=1,2$. For each $l \in \mathbb{Z}^{n}$, let $\psi_{l} \in C_{0}^{\infty}\left(\mathbb{R}^{n}\right)$ such that $\psi_{l}(x)=1$ for $|x| \leq|l| / 4, \psi_{l}(x)=0$ for $|x| \geq|l| / 3,0 \leq \psi(x) \leq 1$, and $\left|\nabla \psi_{l}(x)\right| \leq 20 /|l|$ for any $x \in \mathbb{R}^{n}$. Note that for any $U_{\varepsilon, T_{l}} \in \mathcal{M}\left(T_{l}\right)$,

$$
0 \leq U_{\varepsilon, T_{l}}=\psi_{l} U_{\varepsilon, T_{l}}+\psi_{l}(\cdot-l) U_{\varepsilon, T_{l}}+\left(1-\psi_{l}-\psi_{l}(\cdot-l)\right) U_{\varepsilon, T_{l}}
$$

By $5^{\circ}$ of Theorem 2.2 and (2.3), there exist constants $C_{1}, c>0$ such that for all large $|l|$,

$$
J_{\varepsilon}\left(U_{\varepsilon, T_{l}}\right) \geq J_{\varepsilon}\left(\psi_{l} U_{\varepsilon, T_{l}}\right)+J_{\varepsilon}\left(\psi_{l}(\cdot-l) U_{\varepsilon, T_{l}}\right)-C_{1} \exp (-c|l|) .
$$

For large $|l|$, we see that

$$
\psi_{l} U_{\varepsilon, T_{l}} \in \Gamma\left(T_{1}\right) \quad \text { and } \quad \psi_{l}(\cdot-l) U_{\varepsilon, T_{l}} \in \Gamma\left(l+T_{2}\right)
$$

Thus (2.4)-(2.5) imply that for large $|l|$,

$$
c_{\varepsilon}\left(T_{l}\right) \geq c_{\varepsilon}\left(T_{1}\right)+c_{\varepsilon}\left(T_{2}\right)-C_{1} \exp (-c|l|) .
$$

Now to get $1^{o}$ of Theorem 1.2 , take $U_{\varepsilon, T_{i}} \in \mathcal{M}_{\varepsilon}\left(T_{i}\right)$ for each $i=1,2$. Then for large $|l|$,

$$
u_{\varepsilon, T_{l}} \equiv \psi_{l} U_{\varepsilon, T_{1}}+\psi_{l}(\cdot-l) U_{\varepsilon, T_{2}}(\cdot-l) \in \Gamma\left(T_{l}\right)
$$


Then, again by $5^{\circ}$ of Theorem 2.2 , there exist constants $C_{2}, c>0$ such that for large $|l|$,

$$
\begin{aligned}
c_{\varepsilon}\left(T_{l}\right) & \leq J_{\varepsilon}\left(u_{\varepsilon, T_{l}}\right) \\
& \leq J_{\varepsilon}\left(\psi_{l} U_{\varepsilon, T_{1}}\right)+J_{\varepsilon}\left(\psi_{l}(\cdot-l) U_{\varepsilon, T_{2}}(\cdot-l)\right)+C_{2} \exp (-c|l|) \\
& =J_{\varepsilon}\left(\psi_{l} U_{\varepsilon, T_{1}}\right)+J_{\varepsilon}\left(\psi_{l} U_{\varepsilon, T_{2}}\right)+C_{2} \exp (-c|l|) \\
& \leq J_{\varepsilon}\left(U_{\varepsilon, T_{1}}\right)+J_{\varepsilon}\left(U_{\varepsilon, T_{2}}\right)+2 C_{2} \exp (-c|l|) \\
& =c_{\varepsilon}\left(T_{1}\right)+c_{\varepsilon}\left(T_{2}\right)+2 C_{2} \exp (-c|l|) .
\end{aligned}
$$

Combining (2.6) and (2.7), we get

$$
\lim _{|l| \rightarrow \infty} c_{\varepsilon}\left(T_{l}\right)=c_{\varepsilon}\left(T_{1}\right)+c_{\varepsilon}\left(T_{2}\right) .
$$

To complete the proof of Theorem 1.2, note that if $U_{\varepsilon, T_{l}} \in \mathcal{M}_{\varepsilon}\left(T_{l}\right)$, for large $|l|, \psi_{l} U_{\varepsilon, T_{l}} \in \Gamma\left(T_{1}\right)$ and $\psi_{l}(\cdot-l) U_{\varepsilon, T_{l}} \in \Gamma\left(l+T_{2}\right)$. Then arguing as in (2.7), we get

$$
\lim _{|l| \rightarrow \infty} J_{\varepsilon}\left(\psi_{l} U_{\varepsilon, T_{l}}\right)=c_{\varepsilon}\left(T_{1}\right)
$$

and

$$
\lim _{|l| \rightarrow \infty} J_{\varepsilon}\left(\psi_{l}(\cdot-l) U_{\varepsilon, T_{l}}\right)=\lim _{|i| \rightarrow \infty} J_{\varepsilon}\left(\psi_{l} U_{\varepsilon, T_{l}}(\cdot+l)\right)=c_{\varepsilon}\left(T_{2}\right) .
$$

Lastly, (2.9)-(2.10), the unform boundedness of $\left\{\left.|| U_{\varepsilon, T_{l}}\right|_{C^{2, \alpha}\left(\mathbb{R}^{n}\right)}\right\}$ for any fixed $\alpha \in(0,1)$, and the decay property $5^{\circ}$ of Theorem 2.2 yield $2^{\circ}, 3^{\circ}$ of Theorem 1.2.

\section{Mountain pass results}

In [10], for each finite $T \subset Z^{n}$ and each small $\varepsilon>0$, the existence of a solution, $V_{\varepsilon, T}$, of (1.1) of mountain pass type was proved. This solution satisfies $0<V_{\varepsilon, T}<U_{\varepsilon, T}$ where $U_{\varepsilon, T} \in \mathcal{M}_{\varepsilon}(T)$. In this section, we will obtain a refinement of that result which provides two mountain pass solutions when $T=T_{l}$ with $l$ large. To begin, we recall some results from $\S 3$ of [10].

Let $S \subset T \subset \mathbb{Z}^{n}$ with $T$ finite and $S \neq T$. Define the family of homotopies

$$
\begin{gathered}
\mathcal{G}_{\varepsilon}(S, T) \equiv\left\{g \in C\left([0,1], W^{1,2}\left(\mathbb{R}^{n}\right)\right) \mid U_{\varepsilon, S} \leq g(\theta) \leq U_{\varepsilon, T}\right. \\
\text { and } \left.g(0)=U_{\varepsilon, S}, g(1)=U_{\varepsilon, T}\right\}
\end{gathered}
$$


and define

$$
b_{\varepsilon}(S, T)=\inf _{g \in \mathcal{G}_{\varepsilon}(S, T)} \max _{\theta \in[0,1]} J_{\varepsilon}(g(\theta)) .
$$

Then by Propositions 3.1-3.2 of [10], we have

Proposition 3.1. There are constants, $0<\underline{\beta}<\bar{\beta}<\infty$ such that

$$
\underline{\beta} \leq \liminf _{\varepsilon \rightarrow 0} \sqrt{\varepsilon} b_{\varepsilon}(S, T) \leq \limsup _{\varepsilon \rightarrow 0} \sqrt{\varepsilon} b_{\varepsilon}(S, T)<\bar{\beta} .
$$

With the aid of these propositions, it was proved in [10] that:

Theorem 3.2. Let $S$ and $T$ be as above. Then there is an $\varepsilon_{2}=\varepsilon_{2}(S, T)>0$ such that for any $\varepsilon \in\left(0, \varepsilon_{2}\right)$, there is a solution, $V_{\varepsilon, S, T}$ of (1.1) with $U_{\varepsilon, S}<$ $V_{\varepsilon, S, T}<U_{\varepsilon, T}$ and $J_{\varepsilon}\left(V_{\varepsilon, S, T}\right)=b_{\varepsilon}(S, T)$.

Remark 3.3. Taking $S=T_{1}$ and $T=T_{l}$ yields an $\varepsilon_{2}\left(T_{1}, T_{l}\right)$ and a solution, $V_{\varepsilon, T_{1}, T_{l}}$ of (1.1) with $U_{\varepsilon, T_{1}}<V_{\varepsilon, T_{1}, T_{l}}<U_{\varepsilon, T_{l}}$ for $\varepsilon \in\left(0, \varepsilon_{2}\left(T_{1}, T_{l}\right)\right)$. Similarly taking $S=l+T_{2}$ and $T=T_{l}$ yields an $\varepsilon_{2}\left(l+T_{2}, T_{l}\right)$ and a solution, $V_{\varepsilon,\left(l+T_{2}\right), T_{l}}$ of (1.1) with $U_{\varepsilon, l+T_{2}}<V_{\varepsilon, T_{1}, T_{l}}<U_{\varepsilon, T_{l}}$ for $\varepsilon \in\left(0, \varepsilon_{2}\left(l+T_{2}, T_{l}\right)\right)$.

We seek to show that for large $l$ these two solutions are distinct and then to study their asymptotic behavior as $|l| \rightarrow \infty$. This cannot be done directly from Theorem 3.2 since for $T=T_{l}$ and $S$ as above, it gives an $\varepsilon_{2}$ which depends on $l$. Therefore $\varepsilon_{2}$ may go to 0 as $|l| \rightarrow \infty$. Hence sharper estimates are needed. The proof of Theorem 3.2 requires that

$$
b_{\varepsilon}(S, T)>\max \left(c_{\varepsilon}(S), c_{\varepsilon}(T)\right) .
$$

Thus for our special choices of $S$ and $T=T_{l}$, it suffices to show there is an $\varepsilon^{*}>0$ such that

$$
b_{\varepsilon}\left(S, T_{l}\right)>\max \left(c_{\varepsilon}(S), c_{\varepsilon}\left(T_{l}\right)\right)
$$

holds for all $\varepsilon \in\left(0, \varepsilon^{*}\right)$ and all large $l$. Note that by $(2.6)$, for large $l$,

$$
c_{\varepsilon}\left(T_{l}\right)=\max \left(c_{\varepsilon}(S), c_{\varepsilon}\left(T_{l}\right)\right)
$$

where $S=T_{1}$ or $l+T_{2}$. Hence to obtain (3.4), it suffices to find a constant $\beta_{\varepsilon}$ which is independent of $l$ for $l$ sufficiently large such that for all $\varepsilon \in\left(0, \varepsilon^{*}\right)$,

$$
b_{\varepsilon}\left(S, T_{l}\right)>\beta_{\varepsilon}>c_{\varepsilon}\left(T_{l}\right) .
$$

The following result is useful for that purpose. Let $\sigma>0$ be such that $G^{\prime \prime}(s)>0$ for $s \in[0, \sigma]$. 
Proposition 3.7. Let $\mathcal{D} \subset \mathbb{R}^{n}$ be an open set with a piece-wise smooth boundary and suppose $u \in W^{1,2}\left(\mathbb{R}^{n}\right)$ with $0 \leq u \leq \sigma$ on $\partial \mathcal{D}$. Set

$$
\mathcal{F}(u ; \mathcal{D}) \equiv\left\{\varphi \in W^{1,2}\left(\mathbb{R}^{n}\right) \mid \varphi=u \text { in } \mathbb{R}^{n} \backslash \mathcal{D}\right\} .
$$

Then there exists a unique $w=u_{\mathcal{D}} \in \mathcal{F}(u ; \mathcal{D})$ with $0<w<\sigma$ in $\mathcal{D}$ such that

$$
I_{\mathcal{D}}(w) \equiv \int_{\mathcal{D}} L_{\varepsilon}(w) d x=\inf _{\varphi \in \mathcal{F}(u ; \mathcal{D})} \int_{\mathcal{D}} L_{\varepsilon}(\varphi) d x
$$

Moreover $w$ is a solution of (1.1) in $\mathcal{D}$.

Proof: Let $\left(u_{k}\right)$ be a minimizing sequence for $I_{\mathcal{D}}$. Then $\left(\left\|\nabla u_{k}\right\|_{L^{2}(\mathcal{D})}\right)$ is bounded. Moreover replacing $u_{k}$ by $\zeta_{k}=\min \left(\max \left(u_{k}, 0\right), 1\right)$ for which $I_{\mathcal{D}}\left(\zeta_{k}\right) \leq I_{\mathcal{D}}\left(u_{k}\right)$, it can be assumed that $0 \leq u_{k} \leq 1$. Hence $\left(u_{k}\right)$ is bounded in $W_{l o c}^{1,2}(\mathcal{D})$ and the local weak lower semicontinuity of $I_{\mathcal{D}}$ implies that there is a $w \in \mathcal{F}(u ; \mathcal{D})$ such that along a subsequence, $u_{k} \rightarrow w$ weakly in $W_{l o c}^{1,2}(\mathcal{D})$ and (3.8) holds. To see that $0<w<\sigma$ in $\mathcal{D}$ and therefore by standard elliptic regularity arguments in the calculus of variations, $w=u_{\mathcal{D}}$ is a solution of (1.1) in $\mathcal{D}$, we modify an argument from the proof of Theorem 3.1 of [9]. Since $G$ is even about $1 / 2$, setting $q\left(u_{k}\right)(x)=u_{k}(x)$ if $u_{k}(x) \in[0,1 / 2]$ and $q\left(u_{k}\right)(x)=1-u_{k}(x)$ if $u_{k}(x) \in[1 / 2,1]$ shows $I_{\mathcal{D}}\left(q\left(u_{k}\right)\right) \leq I_{\mathcal{D}}\left(u_{k}\right)$. Therefore replacing $u_{k}$ by $q\left(u_{k}\right)$ if need be, it can be assumed that $0 \leq u_{k} \leq 1 / 2$ and $w$ satisfies the same inequalities. Next set $p\left(u_{k}\right)=\min \left(u_{k}, \sigma\right)$. Then since $G\left(p\left(u_{k}\right)\right) \leq G\left(u_{k}\right)$ and $\nabla p\left(u_{k}\right)=0$ if $u_{k}>\sigma, I_{\mathcal{D}}\left(p\left(u_{k}\right)\right) \leq I_{\mathcal{D}}\left(u_{k}\right)$ so it can be assumed that $0 \leq u_{k} \leq \sigma$ and likewise for $w$. To get the uniqueness, note that if $w$ and $\hat{w}$ are minimizers,

$$
\begin{aligned}
0 & =\int_{\mathcal{D}}\left(-\Delta(w-\hat{w})+A_{\varepsilon}\left(G^{\prime}(w)-G^{\prime}(\hat{w})\right)\right)(w-\hat{w}) d x \\
& =\int_{\mathcal{D}}|\nabla w-\hat{w}|^{2}+A_{\varepsilon} G^{\prime \prime}(z)(w-\hat{w})^{2} d x,
\end{aligned}
$$

where $z$ lies between $w$ and $\hat{w}$. Then, since $G^{\prime \prime}(s)>0$ for $s \in[0, \sigma]$, we get $w \equiv \hat{w}$ in $\mathcal{D}$.

Now to find $\beta_{\varepsilon}$, let $g \in \mathcal{G}_{\varepsilon}\left(S, T_{l}\right)$. Since the argument is the same for either choice of $S$, let $S=T_{1}$. Choose a $\sigma>0$ for which Proposition 3.7 is valid. Let $N_{r}(Q)$ denote an open $r$ neighborhood of $Q$. By $5^{\circ}$ of Theorem 2.2, for all $|l|=|l(\sigma)|$ sufficiently large, $|g(\theta)(x)| \leq \sigma$ for $x \in \mathbb{R}^{n} \backslash N_{|l| / 5}\left(T_{l}\right)$ and 
each $\theta \in[0,1]$. Using the existence result of Proposition 3.7, we define

$$
\hat{g}(\theta)(x)=\left\{\begin{array}{l}
g(\theta)_{\mathbb{R}^{n} \backslash N_{|l| / 5}\left(T_{l}\right)}(x) \text { for } x \in \mathbb{R}^{n} \backslash N_{|l| / 5}\left(T_{l}\right) \\
g(\theta)(x) \text { for } x \in N_{|l| / 5}\left(T_{l}\right) .
\end{array}\right.
$$

Due to the uniqueness result of Proposition 3.7, $\hat{g} \in \mathcal{G}_{\varepsilon}\left(T_{1}, T_{l}\right)$, and because of its definition, $J_{\varepsilon}(\hat{g}(\theta)) \leq J_{\varepsilon}(g(\theta))$. Choose a function $\phi_{l} \in C^{\infty}\left(\mathbb{R}^{n} ;[0,1]\right)$ such that $\phi_{l}(x)=1$ for $x \in N_{|l| / 2}\left(l+T_{2}\right), \phi_{l}(x)=0$ for $x \notin N_{3|l| / 4}\left(l+T_{2}\right)$ and $\left|\nabla \phi_{l}\right| \leq 10 /|l|$. Then define $\tilde{g}(\theta) \equiv \hat{g}(\theta) \phi_{l}$. We see from the decay property $5^{\circ}$ of Theorem 2.2 that there exist constants $D, d>0$ such that for any $x \in \mathbb{R}^{n} \backslash N_{|l| / 5}\left(T_{l}\right), \hat{g}(\theta)(x) \leq D \exp (-d|l|)$. Since

$$
-\Delta \hat{g}(\theta)+A_{\varepsilon}(x) G^{\prime}(\hat{g}(\theta))=0 \text { in } \mathbb{R}^{n} \backslash N_{|l| / 5}\left(T_{l}\right),
$$

by standard local elliptic estimates [14], there exist constants $C^{\prime}, c^{\prime}>0$ such that for any $x \in N_{3|l| / 4}\left(l+T_{2}\right) \backslash N_{|l| / 2}\left(l+T_{2}\right),|\nabla \hat{g}(\theta)(x)| \leq C^{\prime} \exp \left(-c^{\prime}|l|\right)$. Thus, there are constants, $c, C>0$, independent of large $|l|$ and $g \in \mathcal{G}_{\varepsilon}\left(T_{1}, T_{l}\right)$ such that

$$
J_{\varepsilon}(g(\theta)) \geq \int_{N_{3|l| / 4}\left(l+T_{2}\right)} L_{\varepsilon}(\hat{g}(\theta)) d x \geq J_{\varepsilon}(\tilde{g}(\theta))-C \exp (-c|l|) .
$$

Now we define $h \in C\left([0,1], W^{1,2}\left(\mathbb{R}^{n}\right)\right)$ by

$$
h(\theta)(x)=\left\{\begin{array}{l}
3 \theta \min \left\{\tilde{g}(0)(x), U_{\varepsilon, l+T_{2}}(x)\right\} \text { for } \theta \in[0,1 / 3] \\
\min \left\{\tilde{g}(3 \theta-1)(x), U_{\varepsilon, l+T_{2}}(x)\right\} \text { for } \theta \in[1 / 3,2 / 3] \\
(3 \theta-2) U_{\varepsilon, l+T_{2}}(x)+(3-3 \theta) h(2 / 3)(x) \text { for } \theta \in(2 / 3,1] .
\end{array}\right.
$$

Hence $h \in \mathcal{G}_{\varepsilon}\left(\emptyset, l+T_{2}\right)$. Since $U_{\varepsilon, l+T_{2}}(x)=U_{\varepsilon, T_{2}}(x-l)$ and $\left\{J_{\varepsilon}\left(U_{\varepsilon, T_{2}}\right)\right\}$ is uniformly bounded for small $\varepsilon>0$, taking $|l|$ large shows there is a constant, $C_{1}>0$, independent of small $\varepsilon>0$ and large $|l|>0$, such that

$$
\max _{\theta \in[0,1] \backslash[1 / 3,2 / 3]} J_{\varepsilon}(h(\theta)) \leq C_{1},
$$

and

$$
\max _{\theta \in[1 / 3,2 / 3]} J_{\varepsilon}(h(\theta)) \leq \max _{\theta \in[0,1]} J_{\varepsilon}(\tilde{g}(\theta))+C_{1} .
$$


Consequently by the form of $h,(3.9)-(3.11)$, and Proposition 3.1, there is a constant $C_{2}>0$, independent of small $\varepsilon$ and large $l$ such that

$$
\begin{aligned}
\max _{\theta \in[0,1]} J_{\varepsilon}(\tilde{g}(\theta)) & \geq \max _{\theta \in[0,1]} J_{\varepsilon}(h(\theta))-C_{2} \geq b_{\varepsilon}\left(\emptyset, l+T_{2}\right)-C_{2} \\
& =b_{\varepsilon}\left(\emptyset, T_{2}\right)-C_{2} \geq \underline{\beta} / \sqrt{\varepsilon}-C_{2} .
\end{aligned}
$$

Since $\left\{c_{\varepsilon}\left(T_{l}\right) \mid l \in \mathbb{Z}^{n}\right\}$ is bounded, taking $\beta_{\varepsilon}=\beta / \sqrt{\varepsilon}-C_{2}-1$, we conclude that for large $l>0$,

$$
b_{\varepsilon}\left(T_{1}, T_{l}\right)=\inf _{g \in \mathcal{G}_{\varepsilon}\left(T_{1}, T_{l}\right)} \max _{\theta \in[0,1]} J_{\varepsilon}(g(\theta)) \geq \beta_{\varepsilon}-1>c_{\varepsilon}\left(T_{l}\right) .
$$

As a consequence of the above observations, we have:

Corollary 3.13. There is an $r_{0}>0$ and $\varepsilon^{*}=\varepsilon^{*}\left(T_{1}, T_{2}\right)>0$ such that for $|l| \geq r_{0}, \varepsilon \in\left(0, \varepsilon^{*}\right)$, and $S=T_{1}$ or $S=l+T_{2}, b_{\varepsilon}\left(S, T_{l}\right)$ is a critical value of $J_{\varepsilon}$ defined on $\mathcal{G}_{\varepsilon}\left(S, T_{l}\right)$.

Next the asymptotic behavior as $l \rightarrow \infty$ of $b_{\varepsilon}\left(S, T_{l}\right)$ and the corresponding critical points of $J_{\varepsilon}$ will be studied.

Theorem 3.14. Let $A_{\varepsilon}$ and $G$ be as above. Let $U_{\varepsilon, T_{1}}, U_{\varepsilon, T_{2}}$ be respectively the largest members of $\mathcal{M}_{\varepsilon}\left(T_{1}\right), \mathcal{M}_{\varepsilon}\left(T_{2}\right)$ and $U_{\varepsilon, T_{l}}$ be the smallest member of $\mathcal{M}_{\varepsilon}\left(T_{l}\right)$. Then there is an $\varepsilon_{2}=\varepsilon_{2}\left(T_{1}, T_{2}\right) \in\left(0, \varepsilon^{*}\right)$ such that for any $\varepsilon \in\left(0, \varepsilon_{2}\right)$, as $l \rightarrow \infty$,

$1^{o} \quad b_{\varepsilon}\left(T_{1}, T_{l}\right) \rightarrow c_{\varepsilon}\left(T_{1}\right)+b_{\varepsilon}\left(T_{2}\right)$,

$2^{o} V_{\varepsilon, T_{1}, T_{l}} \rightarrow U_{\varepsilon, T_{1}}$ and there is a solution, $V_{\varepsilon, 2}$, of (1.1) with $J_{\varepsilon}\left(V_{\varepsilon, 2}\right)=$ $b_{\varepsilon}\left(T_{2}\right)$ such that $V_{\varepsilon, T_{1}, T_{l}}(\cdot-l) \rightarrow V_{\varepsilon, 2}$, convergence being along a subsequence in $C_{\text {loc }}^{2}$.

$3^{o} b_{\varepsilon}\left(l+T_{2}, T_{l}\right) \rightarrow b_{\varepsilon}\left(T_{1}\right)+c_{\varepsilon}\left(T_{2}\right)$.

$4^{o}$ There is a solution, $V_{\varepsilon, 1}$, of (1.1) with $J_{\varepsilon}\left(V_{\varepsilon, 1}\right)=b_{\varepsilon}\left(T_{1}\right)$ such that $V_{\varepsilon, l+T_{2}, T_{l}} \rightarrow V_{\varepsilon, 1}$ and $V_{\varepsilon, T_{l}}(\cdot-l) \rightarrow U_{\varepsilon, T_{2}}$, convergence being along a subsequence in $C_{l o c}^{2}$.

In particular, by $1^{o}-4^{o}, V_{\varepsilon, T_{1}, T_{l}} \neq V_{\varepsilon, l+T_{2}, T_{l}}$ for large $l$.

Remark 3.15. By $1^{o}-4^{o}, V_{\varepsilon, T_{1}, T_{l}} \neq V_{\varepsilon, l+T_{2}, T_{l}}$ for large $l$, i.e. we have two distinct solutions of (1.1) of mountain pass type.

Proof of Theorem 3.14: We will prove $1^{\circ}-2^{o}$. The remaining items are proved in the same way. Set $p(\theta)=\theta U_{\varepsilon, T_{l}}+(1-\theta) U_{\varepsilon, T_{1}}$ for $\theta \in[0,1]$ so 
$p \in \mathcal{G}_{\varepsilon}\left(T_{1}, T_{l}\right)$. Then there is a constant $M=M(\varepsilon)$ which is independent of large $l$ such that

$$
J_{\varepsilon}(p(\theta)) \leq M(\varepsilon)
$$

for $\theta \in[0,1]$. Hence by $(3.16)$,

$$
b_{\varepsilon}\left(T_{1}, T_{l}\right) \leq M(\varepsilon) .
$$

Now we argue somewhat as in the proof of (3.6). Choose any $\sigma$ for which Proposition 3.7 is valid. For any $g_{l} \in \mathcal{G}_{\varepsilon}\left(T_{1}, T_{l}\right)$, we see that if $|l|>0$ is large, $\left|g_{l}(\theta)(x)\right| \leq \sigma$ for $x \in \mathbb{R}^{n} \backslash N_{|l|} / 10\left(T_{l}\right)$ and each $\theta \in[0,1]$. Using the existence result of Proposition 3.7, define

$$
\hat{g}_{l}(\theta)(x)=\left\{\begin{array}{l}
g_{l}(\theta)_{\mathbb{R}^{n} \backslash N_{|l| / 10}\left(T_{l}\right)}(x) \text { for } x \in \mathbb{R}^{n} \backslash N_{|l| / 10}\left(T_{l}\right) \\
g_{l}(\theta)(x) \text { for } x \in N_{|l| / 10}\left(T_{l}\right) .
\end{array}\right.
$$

The uniqueness result of Proposition 3.7 implies that $\hat{g}_{l} \in \mathcal{G}_{\varepsilon}\left(T_{1}, T_{l}\right)$. Choose a function $\psi_{l} \in C^{\infty}\left(\mathbb{R}^{n} ;[0,1]\right)$ such that $\psi_{l}(x)=1$ for $x \in N_{|l| / 8}\left(T_{l}\right), \psi_{l}(x)=$ 0 for $x \notin N_{|l| / 4}\left(T_{l}\right)$ and $\left|\nabla \psi_{l}\right| \leq 10 /|l|$ and define $\tilde{g}_{l}(\theta) \equiv \hat{g}_{l}(\theta) \psi_{l}$. As in (3.9), we find constants, $c, C>0$, independent of large $|l|>0$ such that

$$
J_{\varepsilon}\left(g_{l}(\theta)\right) \geq J_{\varepsilon}\left(\hat{g}_{l}(\theta)\right) \geq J_{\varepsilon}\left(\tilde{g}_{l}(\theta)\right)-C \exp (-c|l|) .
$$

With $\chi_{S}$ denoting the characteristic function of $S$ as in $\S 2$, define

$$
\tilde{g}_{l, 1}(\theta) \equiv \tilde{g}_{l}(\theta) \chi_{N_{|l| / 2}\left(T_{1}\right)}, \quad \tilde{g}_{l, 2}(\theta) \equiv \tilde{g}_{l}(\theta) \chi_{N_{|l| / 2}\left(l+T_{2}\right)},
$$

Then, by (3.18),

$$
J_{\varepsilon}\left(g_{l}(\theta)\right) \geq J_{\varepsilon}\left(\tilde{g}_{l, 1}(\theta)\right)+J_{\varepsilon}\left(\tilde{g}_{l, 2}(\theta)\right)-C \exp (-c|l|) .
$$

Next define $h_{l}$ as follows:

$$
h_{l}(\theta)(x)=\left\{\begin{array}{l}
3 \theta \min \left\{\tilde{g}_{l, 2}(0)(x), U_{\varepsilon, l+T_{2}}(x)\right\} \text { for } \theta \in[0,1 / 3] \\
\min \left\{\tilde{g}_{l, 2}(3 \theta-1)(x), U_{\varepsilon, l+T_{2}}(x)\right\} \text { for } \theta \in[1 / 3,2 / 3] \\
(3 \theta-2) U_{\varepsilon, l+T_{2}}(x)+(3-3 \theta) h_{l}(2 / 3)(x) \text { for } \theta \in(2 / 3,1] .
\end{array}\right.
$$

Since $h_{l} \in \mathcal{G}_{\varepsilon}\left(\emptyset, l+T_{2}\right)$, Proposition 3.1 shows that

$$
\max _{\theta \in[0,1]} J_{\varepsilon}\left(h_{l}(\theta)\right) \geq b_{\varepsilon}\left(\emptyset, l+T_{2}\right)=b_{\varepsilon}\left(\emptyset, T_{2}\right) \geq \underline{\beta} / \sqrt{\varepsilon} .
$$


Theorem 1.2 and the form of $h_{l}$ show that for some constant $C_{1}>0$, independent of small $\varepsilon>0$ and large $|l|>0$,

$$
\left.\max _{\theta \in[0,1] \backslash(1 / 3,2 / 3)} J_{\varepsilon}\left(h_{l}\right)(\theta)\right) \leq C_{1}
$$

and this implies that for small $\varepsilon>0$ and large $l$,

$$
\left.\max _{\theta \in[0,1]} J_{\varepsilon}\left(h_{l}(\theta)\right)=\max _{\theta \in[1 / 3,2 / 3]} J_{\varepsilon}\left(h_{l}\right)(\theta)\right) .
$$

By Theorem 1.2 and the decay property $5^{o}$ of Theorem 2.2 , for any $x \in \mathbb{R}^{n}$, $\tilde{g}_{l, 2}(3 \theta-1)(x) \leq U_{\varepsilon, T_{l}}(x)$ and

$$
\lim _{|l| \rightarrow \infty}\left\|U_{\varepsilon, T_{l}}-U_{\varepsilon, T_{2}}(\cdot-l)\right\|_{C^{1}\left(\operatorname{supp}\left(\tilde{g}_{l, 2}\right)\right)}=0 .
$$

Therefore

$$
\lim _{|l| \rightarrow \infty} \max _{\theta \in[1 / 3,2 / 3]} J_{\varepsilon}\left(h_{l}(\theta)\right)=\lim _{|l| \rightarrow \infty} \max _{\theta \in[0,1]} J_{\varepsilon}\left(\tilde{g}_{l, 2}(\theta)\right) .
$$

Thus, by (3.20)-(3.22),

$$
\lim _{|l| \rightarrow \infty} \max _{\theta \in[0,1]} J_{\varepsilon}\left(\tilde{g}_{l, 2}(\theta)\right) \geq b_{\varepsilon}\left(\emptyset, T_{2}\right)
$$

Note that for each $\theta \in[0,1], \tilde{g}_{l}^{1}(\theta) \in \Gamma\left(T_{1}\right)$ so

$$
J_{\varepsilon}\left(\tilde{g}_{l}^{1}(\theta)\right) \geq c_{\varepsilon}\left(T_{1}\right) .
$$

Thus, combining (3.24) with (3.19) and (3.23), gives

$$
\liminf _{l \rightarrow \infty} b_{\varepsilon}\left(T_{1}, T_{l}\right) \geq c_{\varepsilon}\left(T_{1}\right)+b_{\varepsilon}\left(T_{2}\right) .
$$

To get an upper bound for $b_{\varepsilon}\left(T_{1}, T_{l}\right)$, a gluing argument will be used. Let $p$ be as in (3.16). Note that by the $5^{\circ}$ of Theorem 2.2 and the fact that $U_{\varepsilon, T_{l}}$ and $U_{\varepsilon, T_{1}}$ are solutions of (1.1), there are constants, $C, c>0$ such that

$$
|p(x)|+|\nabla p(x)| \leq C \exp \left(-c d\left(x, T_{l}\right)\right) .
$$

Let $\delta>0$ and choose $h \in \mathcal{G}_{\varepsilon}\left(\emptyset, l+T_{2}\right)$ such that

$$
\max _{\theta \in[0,1]} J_{\varepsilon}(h(\theta)) \leq b_{\varepsilon}\left(T_{2}\right)+\delta .
$$


Roughly speaking, we would like to glue $p$ restricted to a neighborhood of $T_{1}$ to $h$ restricted to a neighborhood of $l+T_{2}$ and use the resulting function to get the upper bound for $b_{\varepsilon}\left(T_{1}, T_{l}\right)$. However there are some technical problems in doing so because $h$ need not have good enough decay properties and $p(0)=U_{\varepsilon, T_{1}} \neq h(0)$. To get around these difficulties, using Proposition 3.7, set

$$
\hat{h}(\theta)(x)=\left\{\begin{array}{l}
h_{\mathbb{R}^{n} \backslash N_{|l| / 4}\left(l+T_{2}\right)}(\theta)(x) \text { for } x \in \mathbb{R}^{n} \backslash N_{|l| / 4}\left(l+T_{2}\right) \\
h(\theta)(x) \text { for } x \in N_{|l| / 4}\left(l+T_{2}\right)
\end{array}\right.
$$

Then due to the properties of $h_{\mathbb{R}^{n} \backslash N_{|l| / 4}\left(l+T_{2}\right)}$, by (3.27),

$$
\max _{\theta \in[0,1]} J_{\varepsilon}(\hat{h}(\theta)) \leq b_{\varepsilon}\left(T_{2}\right)+\delta
$$

Set $h^{*}(\theta)(x)=\max \left(\hat{h}(\theta)(x), U_{\varepsilon, T_{1}}(x)\right)$ so $h^{*}(0)=U_{\varepsilon, T_{1}}$ and for $l$ large, by $(3.28)$,

$$
\max _{\theta \in[0,1]} \int_{N_{l \mid / 2}\left(l+T_{2}\right)} L_{\varepsilon}\left(h^{*}(\theta)\right) \leq b_{\varepsilon}\left(T_{2}\right)+2 \delta
$$

Choose $r \in(0,|l|)$ with $r$ large enough so that $\left.g\right|_{\partial N_{r}\left(T_{1}\right)}<\sigma$ where $\sigma$ is as in Proposition 3.7. Define $f \in \mathcal{G}_{\varepsilon}\left(T_{1}, T_{l}\right)$ by

$$
f(\theta)(x)=\left\{\begin{array}{l}
p(\theta)(x) \text { for } x \in N_{2 r}\left(T_{1}\right) \\
q(\theta)(x) \text { for } x \in \mathbb{R}^{n} \backslash N_{2 r}\left(T_{1}\right) \cup N_{|l| / 2}\left(l+T_{2}\right) \\
h^{*}(\theta)(x) \text { for } x \in N_{|l| / 2}\left(l+T_{2}\right)
\end{array}\right.
$$

where $q(\theta)$, as given by Proposition 3.7, extends the function whose restriction to $N_{2 r}\left(T_{1}\right)$ is $p(\theta)$ and whose restriction to $N_{|l| / 2}\left(l+T_{2}\right)$ is $h^{*}(\theta)$. Then as earlier, for some constants, $C^{*}, c^{*}>0$,

$$
J_{\varepsilon}(f(\theta)) \leq \int_{N_{2 r}\left(T_{1}\right)} L_{\varepsilon}(p(\theta)) d x+\int_{N_{\frac{|l|}{2}}\left(l+T_{2}\right)} L_{\varepsilon}\left(h^{*}(\theta)\right) d x+C^{*} \exp \left(-c^{*} r\right)
$$

Observe that for fixed $r$, as $|l| \rightarrow \infty$,

$$
\int_{N_{r}\left(T_{1}\right)} L_{\varepsilon}(p(\theta)) d x \rightarrow \int_{N_{r}\left(T_{1}\right)} L_{\varepsilon}\left(U_{\varepsilon, T_{1}}\right) d x \leq c_{\varepsilon}\left(T_{1}\right)
$$


uniformly in $\theta \in[0,1]$. Therefore by (3.29) and (3.31), for large $l$,

$$
J_{\varepsilon}(f(\theta)) \leq \int_{N_{2 r}\left(T_{1}\right)} L_{\varepsilon}\left(U_{\varepsilon, T_{1}}\right) d x+b_{\varepsilon}\left(T_{2}\right)+C^{*} \exp \left(-c^{*} r\right)+2 \delta,
$$

so by (3.31)-(3.32),

$$
\limsup _{l \rightarrow \infty} b_{\varepsilon}\left(T_{1}, T_{l}\right) \leq c_{\varepsilon}\left(T_{1}\right)+b_{\varepsilon}\left(T_{2}\right)+C^{*} \exp \left(-c^{*} r\right)+2 \delta .
$$

Letting $r \rightarrow \infty$, and then $\delta \rightarrow 0$, and combining the result with (3.25) yields $1^{\circ}$ of Theorem 3.14.

To prove $2^{\circ}$ of the Theorem, note first that by Theorem 1.2, as $l \rightarrow$ $\infty, U_{\varepsilon, T_{l}} \rightarrow U \in \mathcal{M}_{\varepsilon}\left(T_{1}\right)$ in $C_{l o c}^{2}\left(\mathbb{R}^{n}\right)$, where $U \geq U_{\varepsilon, T_{1}}$. The choice of $U_{\varepsilon, T_{1}}$ as the largest member of $\mathcal{M}_{\varepsilon}\left(T_{1}\right)$ implies $U=U_{\varepsilon, T_{1}}$. Since $U_{\varepsilon, T_{1}}<V_{\varepsilon, T_{1}, T_{l}}<$ $U_{\varepsilon, T_{l}}$ and $V_{\varepsilon, T_{1}, T_{l}}$ is a solution of (1.1), the first assertion of $2^{o}$ follows. The second requires more work. The uniform bounds for $V_{\varepsilon, T_{1}, T_{l}}$ in $C^{2, \alpha}\left(\mathbb{R}^{n}\right)$, Theorem 1.2, and the choice of $U_{\varepsilon, T_{2}}$ imply there is a solution, $V$, of (1.1) such that $V_{\varepsilon, T_{1}, T_{l}}(\cdot-l) \rightarrow V$ in $C_{l o c}^{2, \alpha}\left(\mathbb{R}^{n}\right)$ along a subsequence as $l \rightarrow \infty$. Therefore with $1<<r<|l| / 2$, estimating as earlier,

$$
\begin{aligned}
& \left|J_{\varepsilon}\left(V_{\varepsilon, T_{1}, T_{l}}\right)-\int_{N_{r}\left(T_{1}\right)} L_{\varepsilon}\left(V_{\varepsilon, T_{1}, T_{l}}\right) d x-\int_{N_{r}\left(T_{2}\right)} L_{\varepsilon}\left(V_{\varepsilon, T_{1}, T_{l}}(\cdot-l)\right) d x\right| \\
& \leq C_{3} \exp (-r) .
\end{aligned}
$$

Hence letting $l$ and then $r \rightarrow \infty,(3.34)$ and $1^{o}$ of this theorem give

$$
c_{\varepsilon}\left(T_{1}\right)+b_{\varepsilon}\left(T_{2}\right)=J_{\varepsilon}\left(U_{\varepsilon, T_{1}}\right)+J_{\varepsilon}\left(V_{\varepsilon, 2}\right)
$$

so

$$
b_{\varepsilon}\left(T_{2}\right)=J_{\varepsilon}\left(V_{\varepsilon, 2}\right)
$$

and Theorem 3.14 is proved.

\section{Some concluding remarks}

Remark 4.1. In $\S 2-\S 3$, we have shown that there are solutions of (1.1) when $T=T_{l}$ corresponding to gluing minima for $T_{1}$ and $l+T_{2}$ and gluing minima for $T_{1}$ to mountain pass solutions for $l+T_{2}$ (as well as the other way around). It is therefore natural to ask whether one can find additional solutions of (1.1) by gluing a mountain pass solution for $T_{1}$ to one for $l+T_{2}$. 
We believe this to be the case. Indeed such solutions were obtained in a related situation in [8] although the question of asymptotic behavior there also remains open.

Remark 4.2. If $T_{1}, T_{2}, \cdots, T_{k}$ are subsets of $\mathbb{Z}^{n}$, one can use the arguments of this note to find solutions of (1.1) corresponding to minima for $T_{1}, l_{2}+$ $T_{2}, \cdots, l_{k}+T_{k}$ provided that the sets $T_{1}, l_{2}+T_{2}, \cdots, l_{k}+T_{k}$ are widely spaced. Similarly one can glue one mountain pass solution to $k-1$ widely separated minima. We expect that there are higher order analogues of these results in the spirit of Remark 4.1.

Remark 4.3. If in Theorem 1.2, $T_{1}$ or $T_{2}$ and hence $T_{l}$ is an infinite set, $c_{\varepsilon}\left(T_{l}\right)$ is infinite. Therefore $1^{o}$ of Theorem 1.2 is not meaningful. However most of $2^{\circ}-3^{o}$ of the theorem can be preserved if the distance between $T_{1}$ and $l+T_{2}, \operatorname{dist}\left(T_{1}, l+T_{2}\right) \rightarrow \infty$ as $l \rightarrow \infty$ for some unbounded set of $l^{\prime} s$. To be more precise, recall that from Theorem 1.1 of [9], if $S \subset \mathbb{Z}^{n}$ is infinite, there is still a solution, $\mathcal{U}_{\varepsilon, S}$, of $(1.1)$ in $\Gamma(S)$. Moreover $\mathcal{U}_{\varepsilon, T}$ is a minimal solution, i.e. for all smooth $\varphi$ having compact support,

$$
\int_{\mathbb{R}^{n}}\left(L_{\varepsilon}\left(\mathcal{U}_{\varepsilon, S}+\varphi\right)-L_{\varepsilon}\left(\mathcal{U}_{\varepsilon, S}\right)\right) d x \geq 0
$$

As was the case in Theorem $2.2, \mathcal{U}_{\varepsilon, S}$ need not be unique. Returning to the current setting, suppose that $S=T_{l}=T_{1} \cup\left(l+T_{2}\right)$ and assume

$\left(l^{*}\right) \mathcal{I} \equiv\left\{l \in \mathbb{Z}^{n} \mid \operatorname{dist}\left(T_{1}, l+T_{2}\right)>0\right\}$ is unbounded.

As a simple example, suppose that $T_{1}$ and $T_{2}$ are infinite subsets of $\mathbb{R}^{n-1} \times$ $\{0\}$. Then we can take $\mathcal{I}=\left\{l_{n} e_{n} \mid l_{n} \in \mathbb{N}\right\}$.

Now we have:

Theorem 4.5. Suppose that $\left(l^{*}\right)$ holds. Then for each $\varepsilon \in\left(0, \varepsilon_{0}\right)$,

$\left(1^{o}\right)$ there is a minimal solution, $\mathcal{U}_{\varepsilon, T_{1}}$ of (1.1) with $\mathcal{U}_{\varepsilon, T_{1}} \in \Gamma\left(T_{1}\right)$ such that along a sequence of $\left(l_{p}\right) \subset \mathcal{I}$ with $l_{p} \rightarrow \infty$ as $p \rightarrow \infty, \mathcal{U}_{\varepsilon, T_{l_{p}}} \rightarrow \mathcal{U}_{\varepsilon, T_{1}}$ in $C_{l o c}^{2}\left(\mathbb{R}^{n}\right)$

$\left(2^{o}\right)$ there is a minimal solution, $\mathcal{U}_{\varepsilon, T_{2}}$ of (1.1) with $\mathcal{U}_{\varepsilon, T_{2}} \in \Gamma\left(T_{2}\right)$ such that along a sequence of $\left(m_{p}\right) \subset \mathcal{I}$ with $m_{p} \rightarrow \infty$ as $p \rightarrow \infty, \mathcal{U}_{\varepsilon, T_{m_{p}}}(\cdot+$ $\left.m_{p}\right) \rightarrow \mathcal{U}_{\varepsilon, T_{2}}$ in $C_{\text {loc }}^{2}\left(\mathbb{R}^{n}\right)$.

Proof: Since $\left\|\mathcal{U}_{\varepsilon, T_{l}}\right\|_{L^{\infty}\left(\mathbb{R}^{n}\right)} \leq 1$ for all $l \in \mathcal{I}$, using the local $W^{k, p}$ and Schauder estimates, as e.g. in [9], shows for any $\alpha \in(0,1)$, there is a constant, $K=K(\alpha)$ such that

$$
\left\|\mathcal{U}_{\varepsilon, T_{l}}\right\|_{C^{2, \alpha}\left(\mathbb{R}^{n}\right)} \leq K(\alpha)
$$


independently of $l \in \mathcal{I}$. Therefore the Arzela-Ascoli Theorem and (1.1) imply the existence of the solution, $\mathcal{U}_{\varepsilon, T_{1}}$, as a limit of $\mathcal{U}_{\varepsilon, T_{l_{p}}}$. That $\mathcal{U}_{\varepsilon, T_{l_{p}}} \in \Gamma\left(T_{l_{p}}\right)$ for all $p \in \mathcal{I}$ implies $\mathcal{U}_{\varepsilon, T_{1}} \in \Gamma\left(T_{1}\right)$. Moreover, as the $C_{\text {loc }}^{2}$ limit of minimal solutions of (1.1), (4.4) shows $\mathcal{U}_{\varepsilon, T_{1}}$ is minimal. A similar argument establishes $2^{\circ}$.

\section{Acknowledgements}

This research of the first author was supported by Mid-career Researcher Program through the National Research Foundation of Korea (NRF) funded by the Ministry of Science, ICT and Future Plannig (No. NRF2013R1A2A2A05006371).

\section{References}

[1] Francesca Alessio, Louis Jeanjean and Piero Montecchiari, Stationary layered solutions in $\mathbb{R}^{2}$ for a class of non autonomous Allen-Cahn equations, Calc. Var. Partial Differential Equations, 11 (2000), 177-2021. MR1782992

[2] Francesca Alessio, Louis Jeanjean and Piero Montecchiari, Existence of infinitely many stationary layered solutions in $\mathbb{R}^{2}$ for a class of periodic Allen-Cahn equations, Comm. Partial Differential Equations, 27 (2002), 1537-1574. MR1924477

[3] Francesca Alessio and Piero Montecchiari, Entire solutions in $\mathbb{R}^{2}$ for a class of Allen-Cahn equations, ESAIM Control Optim. Calc. Var., 11 (2005), 633-672. MR2167878

[4] Francesca Alessio and Piero Montecchiari, Multiplicity of entire solutions for a class of almost periodic Allen-Cahn type equations, Adv. Nonlinear Stud., 5 (2005), 515-549. MR2180581

[5] Victor Bangert, On minimal laminations of the torus, Ann. Inst. Poincare Anal. Non Lineaire, 6 (1989), 95-138. MR0991874

[6] Ugo Bessi, Many solutions of elliptic problems on $\mathbb{R}^{n}$ of irrational slope, Comm. Partial Differential Equations, 30 (2005), 1773-1804. MR2182311

[7] Ugo Bessi, Slope-changing solutions of elliptic problems on $\mathbb{R}^{n}$, Nonlinear Anal., 68 (2008), 3923-3947. MR2416096 
[8] Sergey Bolotin and Paul H. Rabinowitz, Hybrid mountain pass homoclinic solutions of a class of semilinear elliptic PDEs, to appear Ann. Inst. Poincare Anal. Non Lineaire. MR3165281

[9] Jaeyoung Byeon and Paul H. Rabinowitz, On a phase transition model, Calc. Var. Partial Differential Equations, 47 (2013), 1-23. MR3044129

[10] Jaeyoung Byeon and Paul H. Rabinowitz, A note on mountain pass solutions for a class of Allen-Cahn models, to appear in RIMS Koyuroku.

[11] Luis A. Caffarelli and Rafael de la Llave, Planelike minimizers in periodic media. Comm. Pure Appl. Math., 54 (2001), 1403-1441. MR1852978

[12] Rafael de la Llave and Enrico Valdinoci, Multiplicity results for interfaces of Ginzburg-Landau Allen-Cahn equations in periodic media, Adv. Math., 215 (2007), 379-426. MR2354993

[13] Rafael de la Llave and Enrico Valdinoci, A generalization of AubryMather theory to partial differential equations and pseudo-differential equations, Ann. Inst. H. Poincare Anal. Non Lineaire, 26 (2009), 1309 1344. MR2542727

[14] David Gilbarg and Neil S. Trudinger, Elliptic Partial Differential Equations of Second Order, Berlin, Heidelberg, New York and Tokyo, Springer (1983). MR0737190

[15] Jurgen Moser, Minimal solutions of a variational problems on a torus, Ann. Inst. Poincare Anal. Non Lineaire, 3 (1986), 229-272. MR0847308

[16] Matteo Novaga and Enrico Valdinoci, Bump solutions for the mesoscopic Allen-Cahn equation in periodic media. Calc. Var. Partial Differential Equations, 40 (2011), no. 1-2, 37-49. MR2745195

[17] Paul H. Rabinowitz and Ed Stredulinsky, Mixed states for an AllenCahn type equation, Comm. Pure Appl. Math., 56 (2003), 1078-1134. MR1989227

[18] Paul H. Rabinowitz and Ed Stredulinsky, Mixed states for an AllenCahn type equation. II, Calc. Var. Partial Differential Equations, 21 (2004), 157-207. MR2085301

[19] Paul H. Rabinowitz and Ed Stredulinsky, On a class of infinite transition solutions for an Allen-Cahn model equation, Discrete Contin. Dyn. Syst., 21 (2008), 319-332. MR2379469 
[20] Paul H. Rabinowitz and Ed Stredulinsky, Extensions of Moser-Bangert Theory: Locally Minimal Solutions, Progress in Nonlinear Differential Equations and Their Applications, 81, Birkhauser, Boston (2011). MR2809349

[21] Enrico Valdinoci, Plane-like minimizers in periodic media: jet flows and Ginzburg-Landau-type functionals, J. Reine Angew. Math., 574 (2004), 147-185. MR2099113

JAEYOUNG BYEON

Department of Mathematical Sciences, Kaist

291 DaehaK-Ro, Yuseong-Gu, Daejeon 305-701

REPUBLIC OF KOREA

E-mail address: byeon@kaist.ac.kr

PAUl H. RABinowitz

Department of Mathematics

UNIVERSITY OF WISCONSIN-MADISON

MADISON, Wisconsin, 53706

USA

E-mail address: rabinowi@math.wisc.edu

Received August 19, 2013 